\title{
Huge, irreducible femoral hernia interpreted as a preperitoneal lipoma in radiological findings
}

\author{
Paweł Osemek, Krzysztof Paśnik, Piotr Trojanowski \\ Department of General, Oncological and Thoracic Surgery, Military Institute of Health Services, Warsaw, Poland
}

Videosurgery and other miniinvasive techniques 2010; 5 (1): 35-37 DOI: 10.5114/wiitm.2010.13605

\begin{abstract}
Occurrence of pathological masses in the inguinal region, mimicking hernias, is an important surgical and radiological problem. Presence of a hernial sac is the determining factor. Herein, we present a case of irreducible femoral hernia with the greater omentum in its sac, interpreted as a preperitoneal lipoma in ultrasonography and computed tomography depictions. The patient underwent Rutkow tension-free hernioplasty with sac revision and dissection of the greater omentum.
\end{abstract}

Key words: femoral hernia, lipoma, greater omentum, computed tomography.

\section{Introduction}

The incidence of femoral hernias is estimated at approximately $2.3 \%$ of all hernias [1]. It is estimated that they account for approximately $5-10 \%$ of all groin hernias in adults. Most of them occur in women aged over 50 years [2]. A preferable method of inguinal hernia repair nowadays is the use of mesh graft in tension-free open techniques $[3,4]$ as well as laparoscopic repair $[5,6]$.

Unlike the high incidence of spermatic cord or round ligament lipomas, medical reports on an incorrect radiological depiction suggesting a retroperitoneal lipoma as an irreducible femoral hernia containing the greater omentum are relatively rare [7]. All patients suffering from inguinal and femoral hernias are qualified for hernioplasty usually by medical investigation, physical examination and ultrasonography of the inguinal canal and femoral triangle.

Pre-operation herniography, computed tomography scanning (CT) and/or magnetic resonance (MR) imaging are recommended in case of any diagnostic doubts. However, the value and fidelity of the mentioned imaging techniques should not be overestimated. CT and/or USG depiction may differentiate from intraoperational findings $[8,9]$.

\section{Case report}

A 60-year old female was referred to our department for operational treatment of a tumour located in the right inguinal region extending to the right thigh. She had been suffering from this pathology for 25 years. The patient's previous surgical history was negative. A coexisting morbidity was pharmacologically controlled, stable hypertension.

Physical examination revealed a massive irreducible tumour with no signs of pressure on the right femoral nerve and blood vessels. There were no signs of inflammation. The cough impulse was positive. Pulse and feeling were symmetrical on both legs. Motor losses of the right lower extremity were absent. On palpation the abdomen was tender. 
Neither rebound nor local swellings and distensions were present. Laboratory results were normal.

Ultrasonography revealed a tumour without any pathological vascularization, suggesting a picture of lipoma. Preoperational CT scans demonstrated a view of an inguinal hernial sac containing a structure interpreted as a lipoma with the size of $126 \times 90 \mathrm{~mm}$. Dilatation to $26 \mathrm{~mm}$ of the deep inguinal ring was also described (Figure 1).

On the day of admission the patient underwent operation. The right inguinal canal was opened and explored. A large tumour descending down towards the right femoral canal was recognized. The hernial sac was opened and the mass inside, considered as the greater omentum (Figure 2), was resected. Surgical reconstruction was performed with an anterior mesh repair mode Rutkow's procedure. Afterwards the wound was drained for a day.

The postoperative course was uneventful. The patient was discharged home on the next day after the operation. Ambulatory surgical examination in 6 days was recommended. The patient was doing well during the 6 months of follow-up.

\section{Discussion}

Using radiological techniques the main pathological conditions manifesting as masses in the groin fall into

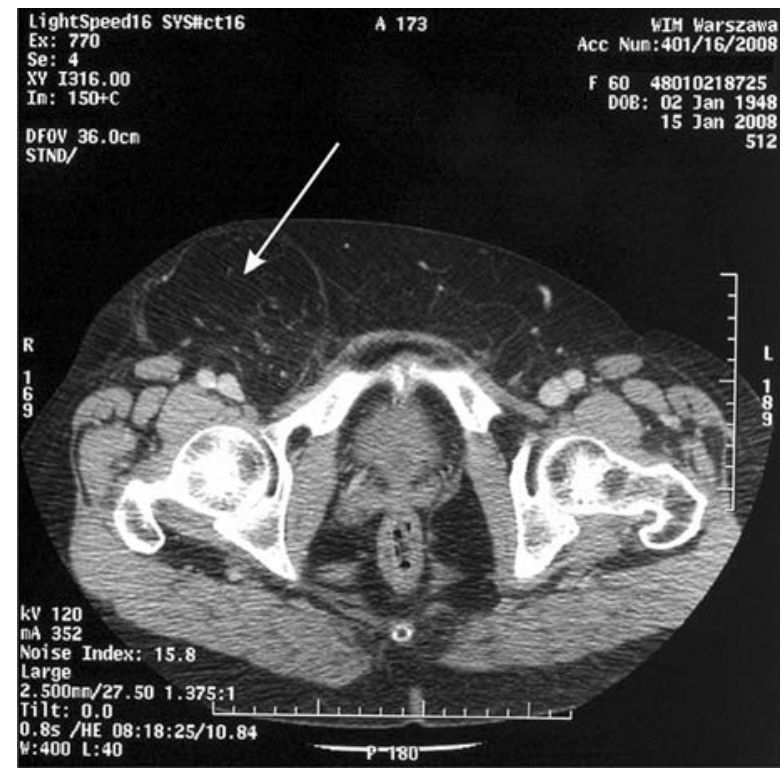

Figure 1. Computed tomographic scan of inguinal "lump" suggesting a preperitoneal lipoma (arrow indicates the "lump") five major groups: congenital abnormalities, noncongenital inguinal and femoral hernias, vascular conditions, infectious or inflammatory processes, and neoplasms [10].

A femoral hernia lies in the femoral canal, medial to the femoral vein. Because of the narrowness of the femoral ring, it is more likely than an inguinal hernia to become incarcerated. Herniography allows the hernial sac with its contents to be outlined. Ultrasonography can usually be performed for direct visualization of bowel loops in peristalsis, motion of peritoneal fluid and echogenic omental fat. These are the most common anatomical elements located in hernial sacs. Congenital inguinal hernias are usually indirect, and they contain bowel loops, omental fat and/or peritoneal fluid. Hernias diagnosed on computed tomography and magnetic resonance imaging are usually incidental discoveries [10].

Haematomas and false aneurysms, usually recognised as complications of femoral artery catheterization, can be located in the femoral triangle region. True aneurysms associated with atherosclerosis of femoral vessels are rarer. Other rarer vascular masses located in this region include varicoceles, varices of the large saphenous vein and post-traumatic arteriovenous fistulas. Some inflammatory processes, such as inflammation of the iliopectineal bursa, synovial osteochondromatosis and abscesses, can result in pathological groin masses.

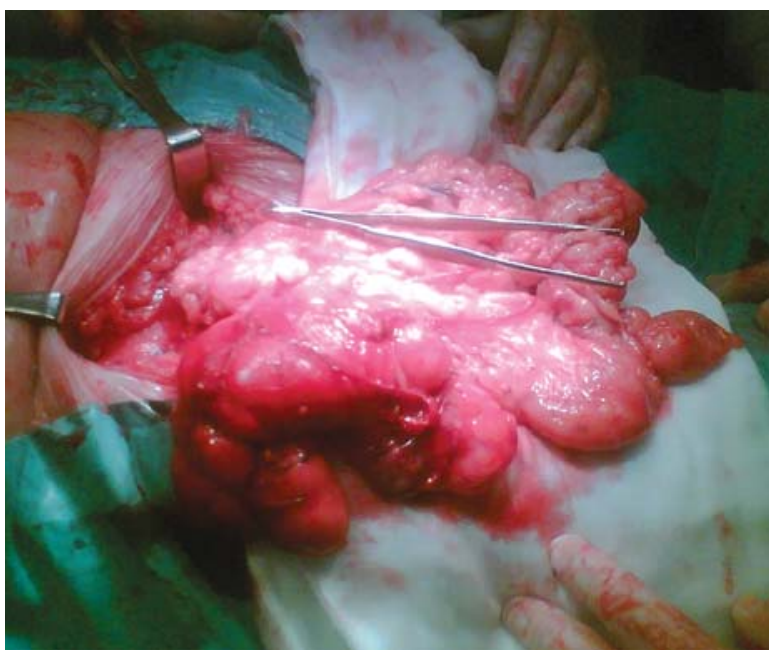

Figure 2. Intraoperational view of right femoral hernia sac containing fragment of omentum mimicking a giant cord lipoma in radiological depictions 
Primary benign tumours of the groin may arise from connective tissue, muscle, fat, blood vessels and lymphoid tissue. Malignant tumours most often involve the inguinal region by direct extension from adjacent structures [10]. They can be primary or metastatic. Some reports mention the occurrence of pseudomyxoma peritonei [8], cholangiocarcinoma metastases [11], malignant mesothelioma [12], liposarcoma of the spermatic cord [13] and endometriosis of the round ligament [146]. Other malignant neoplasms metastasing to inguinal lymph nodes are: melanoma malignum, and primary cancer of the vagina, vulva, penis, anus and rectum, and lower extremities.

The most common benign tumour of the inguinal canal is a lipoma. Cord lipoma is considered to be a continuation of preperitoneal fat. Speed (1914) reported the incidence of cord lipoma, encountered equally with both indirect and direct hernias, to be $47.4 \%$ [15]. The incidence of cord lipoma is currently estimated at $22.5 \%$ of all groin hernias; $23 \%$ of them are associated with indirect and $11 \%$ with direct hernias [7]. Others evaluate the incidence of cord lipomas as $72.5 \%$, which seems high [16]. Lipomas occur more frequently in large hernias (Nyhus type II and IIIb) [16]. Radiological depiction can misidentify it as a real inguinal or femoral hernia, containing a fragment of the greater omentum. The presence of the peritoneal hernial sac determines the nature of the pathology. As early as in 1948 Watson suggested that hypertrophic, inguinal fatty tissue should be considered as hernias only when accompanied by a peritoneal sac. However, sliding lipoma refers to a sliding herniation of preperitoneal fat into the inguinal canal, forming a reducible indirect inguinal hernia in the absence of a peritoneal sac [17]. These should be distinguished from cord lipomas, which present either as an irreducible groin tumour or an incidental finding during open hernioplasty.

\section{Conclusions}

Occasionally, despite significant improvement in imaging capabilities of the groin region, the diagnosis can only be finally determined during an operation.

\section{References}

1. Rutkow IM. Epidemiologic, economic and sociologic aspects of hernia surgery in the Unated States in 1990s. Surg Clin North Am 1998; 78: 941-51.

2. Kingsnorth A, Majid A. Fundamentals of surgical practice. 2nd ed. Cambridge University Press, Cambridge 2006; 279.

3. Amid PK. Lichtenstein tension-free hernia repair: its background and evolution with focus on avoiding postherniorrhaphy chronic pain. Videosurgery and other miniinvasive techniques 2009; 4: 26-31.

4. Mitura K, Romańczuk M, Wróblewski T. Early results of inguinal hernia repair with Desarda method in 17 patients. A preliminary report. Videosurgery and other miniinvasive techniques 2006; 1: 18-22.

5. Czudek S, Skrovina M, Adamcik L. Laparoscopic treatment of inguinal hernia - TOM (transabdominal onlay mesh). Videosurgery and other miniinvasive techniques 2009; 4: 16-9.

6. Michalik M, Frask A, Klawiter A. Inguinal hernia laparoscopic repair. Videosurgery and other miniinvasive techniques 2007; 2: 150-5.

7. Lilly MC, Arregui ME. Lipomas of the cord and round ligament. Ann Surg 2002; 235: 586-90.

8. Shimoyama $S$, Kuramoto $S$, Kawahara $M$, et al. A rare case of pseudomyxoma peritonei presenting an unusual inguinal hernia and splenic metastasis. J Gastroenterol Hepat 2001; 16: 825-9.

9. Ścierski A, Roechte F. The significance of the use of ultrasound in diagnosing inguinal hernia. Videosurgery and other miniinvasive techniques 2007; 2: 58-65.

10. Shadbolt C, Heinze S, Dietrich R. Imaging of groin mases: inguinal anatomy and pathologic conditions revisited. Radiographics 2001; 21: 261-71.

11. Yokoyama N, Shirai Y, Yamazaki H, Hatakeyama K. An inguinal hernia sac tumor of extrahepatic cholangiocarcinoma origin. World J Surg Oncol 2006; 4: 13.

12. Washino S, Terauchi F, Matsuzaki A, Kobayashi Y. Case of malignant mesothelioma presenting as a perineal and intrascrotal mass. Hinyokika Kiyo 2008; 54: 619-23.

13. Soler Soler JL, Zuluaga Gómez A, Hidalgo Domínguez MR, et al. Liposarcoma of the spermatic cord: a report of a new case and a review of the literature. Actas Urol Esp 1999; 23: 447-54.

14. Licheri S, Pisano G, Erdas E, et al. Endometriosis of the round ligament: description of a clinical case and review of the literature. Hernia 2005; 9: 294-7.

15. Read RC, Schaefer RF. Lipoma of the spermatic cord, fatty herniation, liposarcoma. Hernia 2000; 4: 149-54.

16. Carilli S, Alper A, Emre A. Inguinal cord lipomas. Hernia 2004; 8: 252-4.

17. Lau H. Sliding lipoma: an inderect inguinal hernia without a peritoneal sac. J Laparoendosc Adv Surg Tech A 2004; 14: 57-9.

\section{Acknowledgments}

The authors would like to thank the Department of Radiology, Military Institute of Health Services, Warsaw for making radiological images available to us. 